\title{
Role of Osteal Macrophages in Bone Metabolism
}

\author{
Sun Wook Cho \\ Department of Internal Medicine, Seoul National \\ University Hospital, Seoul, Korea \\ Received: January 31, 2015 \\ Accepted: February 2, 2015

\section{Corresponding Author} \\ Sun Wook Cho, M.D., Ph.D. \\ Department of Internal Medicine, Seoul National \\ University Hospital, 101 Daehak-ro, Jongno-gu, \\ Seoul 110-744, Korea \\ Tel: +82-2-2072-2114 \\ Fax: +82-2-762-2292 \\ E-mail: swchomd@gmail.com
}

\begin{abstract}
Macrophages have been shown to have pleiotropic functions in various pathophysiologies, especially in terms of anti-inflammatory and regenerative activity. Recently, the novel functions of bone marrow resident macrophages (called osteal macrophages) were intensively studied in bone development, remodeling and tissue repair processes. This review discusses the current evidence for a role of osteal macrophages in bone modeling, remodeling, and fracture healing processes.
\end{abstract}

Key Words: Osteal macrophage; Bone remodeling; Fracture healing
Macrophages are abundant immune cells in bone marrow. Classically, macrophages are rapidly recruited into infectious or injured sites where they play critical roles in innate immunity. Moreover, macrophages regulate tissue homeostasis in various pathophysiologic processes including innate and adaptive immunity, wound healing, hematopoiesis, and malignancy. For examples, macrophages not only initiate tissue inflammation, but also promote wound healing and tissue remodeling. ${ }^{1} \mathrm{Mac}-$ rophages are schematically classified into two subtypes: classically activated, pro-inflammatory M1-macrophages and alternatively activated, anti-inflammatory/regenerative M2-macrophages. $^{1}$

Apart from osteoclasts, osteal macrophages in bone marrow were recently recounted as a pivotal player in bone metabolism. In murine tissues, F4/80 monoclonal antibody ${ }^{2}$ has been widely used to distinguish mature macrophages from osteoclasts, since F4/80 is rapidly down-regulated in the early stage of osteoclastogenesis. ${ }^{3}$ At the bone and marrow interface, especially in the bone multicellular units, osteal macrophages form a cellular canopy structure over the bone-forming osteoblast. ${ }^{4}$ In particular, the bone remodeling site, which is affected by anabolic factors such as parathyroid hormone (PTH; PTH1-34), showed augmented osteal macrophages (Fig. 1). ${ }^{5}$ These observations suggest that osteal macrophages might play a role in bone remodeling.

\section{OSTEAL MACROPHAGES IN BONE FORMATION}

Evidence suggests that macrophages participate in pleiotropic aspects of bone metabolism. Macrophages have been involved in vascular calcification in ectopic bone formation. Co-culture of macrophages with calcifying vascular cells ${ }^{6}$ or human vascular smooth muscle cells ${ }^{7}$ enhanced alkaline phosphatase activity and mineralization potential. Tumor necrosis factor ${ }^{6,7}$ and oncostatin $\mathrm{M}^{7}$ have been suggested as molecular mediators of macrophage-derived vascular calcification. In addition, depletion of macrophages reduced osteophyte formation in osteoarthritic models, ${ }^{8-10}$ and macrophages have been implicated in the sites of pathologic bone loss in inflammatory bone disorders. ${ }^{11,12}$ These observations suggest that macrophages play a critical role in bone formation and mineralization.

Depletion of macrophages in primary calvarial osteoblast cultures in vitro has been shown to delay osteogenic differentiation and mineralization. ${ }^{4,13}$ In the macrophage fas-induced apoptosis (Mafia) transgenic mouse model, short-term depletion of macrophages with treatment of a synthetic ligand in vivo showed a quantitative reduction of bone formation sites in endocortical bones. ${ }^{4,13}$ In a recent study, in vivo long-term depletion of macrophages in young (3-21 days) and adult (16-22 months) Mafia mice demonstrated an osteopenic phenotypes with suppressed 


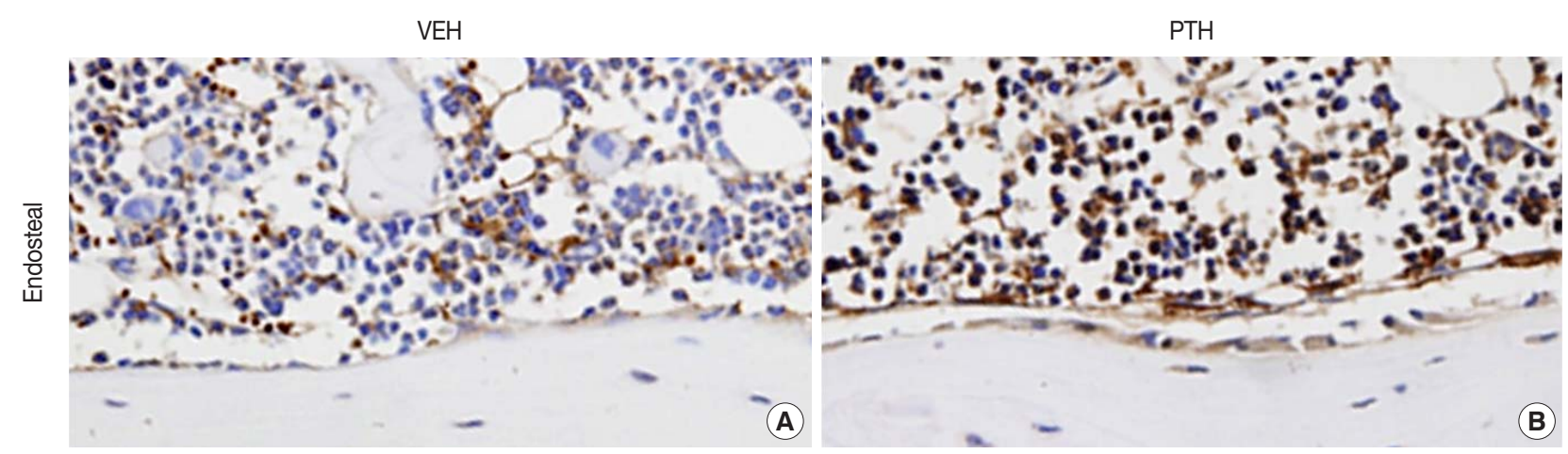

Fig. 1. Osteal macrophages at the bone formation sites of murine bones. F4/80-positive osteal macrophages create a canopy-like structure over the bone remodeling site. Compare to the vehicle (VEH) treatment (A), administration of parathyroid hormones (PTH) enhances bone formation, resulting in cuboidal-changes of osteoblasts and increased recruitment of osteal macrophages at bone remodeling sites (B). ${ }^{5}$

serum bone turnover markers. ${ }^{5}$ The anabolic actions of PTH in bone were markedly reduced in this model. ${ }^{5}$ This study reinforced the hypothesis that osteal macrophages play a pivotal role in bone anabolism. Another independent study with lysozyme M-deficient mice also showed that pre-natal macrophage depletion led to early skeletal growth retardation and progressive osteoporosis. ${ }^{14}$ The latter two studies clearly showed that functional osteoclasts were not significantly affected in these macrophage-deficient models. ${ }^{5,14}$ Taken together, these results suggest that osteal macrophages play an essential role in normal bone development and remodeling, especially through anabolic actions.

One of the critical concerns with osteal macrophages is how to distinguish bone marrow resident macrophages from osteoclasts, since they share the same precursors. Studies of Mafia mice or lysozyme M-deficient mice ${ }^{14}$ showed functionally active osteoclast activities, while macrophages were remarkably depleted, but still there were possible impacts of subtle changes in osteoclasts on overall bone metabolism. A recent study showed more clear evidence supporting the independent presence of functioning osteal macrophages apart from osteoclasts. CCL5deficient mice showed decreased F4/80-positive macrophages at the endocortical bone surface, following reduced bone formation compared to the wild-type mice. ${ }^{15}$ Osteoclastogenesis was enhanced in this model. ${ }^{15}$ More studies with a bone marrowspecific macrophage depletion model are needed.

\section{OSTEAL MACROPHAGES IN BONE REPAIR}

Fracture healing is composed of inflammation and bone repair processes, including endochondral ossification. Previous studies have demonstrated that macrophages are present during multiple stages of fracture healing, and produce mesenchymal growth factors. ${ }^{16}$ Macrophages are also associated with more stable callus formation and healthy union. ${ }^{17}$

Keeping pace with the studies of osteal macrophages in bone metabolism, several groups have extensively studied how osteal macrophages participate in fracture healing of bone. Both systemic and local depletions of macrophages delayed fracture healing and impaired woven bone formation, while treatment of colony stimulating factor 1 increased macrophage recruitment into the injury sites and supported woven bone formation. ${ }^{18}$ This study showed that macrophages were essential for collagen type I-positive matrix formation and bone mineralization. ${ }^{18}$ Similarly, an independent study also showed that depletion of macrophages during fracture repair, even after several days later to fracture, led to impaired bone union with incomplete callus formation accompanied with more fibrotic changes. They observed that macrophages were also involved in promoting the osteogenic differentiation of marrow mesenchymal progenitor cells. ${ }^{14}$ Moreover, a recent study clarified that inflammatory M1-macrophages $\left(\mathrm{F} 4 / 80^{+} \mathrm{Mac}-2^{+}\right)$played a crucial role in the initiation of early inflammation, and both inflammatory ( $\left.\mathrm{F} 4 / 80^{+} \mathrm{Mac}-2^{+}\right)$ and resident ( $\left.\mathrm{F} 4 / 80^{+} \mathrm{Mac}-2^{-}\right)$macrophages derived anabolic signals for endochondral callus formations in murine fracture models. ${ }^{19}$ Taken together, accumulating evidence suggests that macrophages and their specific molecular mediators contribute to fracture healing in a phase-specific polarization-dependent manner.

\section{CONCLUSIONS AND FUTURE DIRECTIONS}

To date, osteal macrophages have been considered a third cellular component, in addition to osteoblasts and osteoclasts. Mac- 
rophages construct a cellular canopy structure over bone remodeling sites, coordinate osteoclast-to-osteoblast coupling, and drive anabolic cytokines for bone formation. Macrophages also create a regenerative microenvironment in the fracture healing processes. In addition, macrophages might play a role in bone and marrow interactions especially at the osteoblastic stem cell niche. Targeting osteal macrophages or their molecular mediators could be potent therapeutic challenges for developing anabolic therapies for bone disease. Further studies are needed to develop specific targets that could be distinguished from osteoclast or inflammatory macrophages.

\section{Conflicts of Interest}

No potential conflict of interest relevant to this article was reported.

\section{REFERENCES}

1. Murray PJ, Wynn TA. Protective and pathogenic functions of macrophage subsets. Nat Rev Immunol 2011; 11: 723-37.

2. Austyn JM, Gordon S. F4/80, a monoclonal antibody directed specifically against the mouse macrophage. Eur J Immunol 1981; 11: 805-15.

3. Lean JM, Matsuo K, Fox SW, et al. Osteoclast lineage commitment of bone marrow precursors through expression of membrane-bound TRANCE. Bone 2000; 27: 29-40.

4. Chang MK, Raggatt LJ, Alexander KA, et al. Osteal tissue macrophages are intercalated throughout human and mouse bone lining tissues and regulate osteoblast function in vitro and in vivo. J Immunol 2008; 181: 1232-44.

5. Cho SW, Soki FN, Koh AJ, et al. Osteal macrophages support physiologic skeletal remodeling and anabolic actions of parathyroid hormone in bone. Proc Natl Acad Sci U S A 2014; 111: 1545-50.

6. Tintut Y, Patel J, Territo M, Saini T, Parhami F, Demer LL. Monocyte/ macrophage regulation of vascular calcification in vitro. Circulation 2002; 105: 650-5.

7. Shioi A, Katagi M, Okuno Y, et al. Induction of bone-type alkaline phosphatase in human vascular smooth muscle cells: roles of tumor necrosis factor-alpha and oncostatin $\mathrm{M}$ derived from macrophages. Circ Res 2002; 91: 9-16.
8. van Lent PL, Blom AB, van der Kraan P, et al. Crucial role of synovial lining macrophages in the promotion of transforming growth factor beta-mediated osteophyte formation. Arthritis Rheum 2004; 50: 103-11.

9. Blom AB, van Lent PL, Holthuysen AE, et al. Synovial lining macrophages mediate osteophyte formation during experimental osteoarthritis. Osteoarthritis Cartilage 2004; 12: 627-35.

10. Kamekura S, Hoshi K, Shimoaka T, et al. Osteoarthritis development in novel experimental mouse models induced by knee joint instability. Osteoarthritis Cartilage 2005; 13: 632-41.

11. Kaneko M, Tomita T, Nakase T, et al. Expression of proteinases and inflammatory cytokines in subchondral bone regions in the destructive joint of rheumatoid arthritis. Rheumatology (Oxford) 2001; 40: 247-55.

12. Haynes DR, Hay SJ, Rogers SD, Ohta S, Howie DW, Graves SE. Regulation of bone cells by particle-activated mononuclear phagocytes. J Bone Joint Surg Br 1997; 79: 988-94.

13. Pettit AR, Chang MK, Hume DA, Raggatt LJ. Osteal macrophages: a new twist on coupling during bone dynamics. Bone 2008; 43: 97682.

14. Vi L, Baht GS, Whetstone H, et al. Macrophages promote osteoblastic differentiation in-vivo: implications in fracture repair and bone homeostasis. J Bone Miner Res 2014 Dec 8 [Epub]. http://dx.doi.org/ 10.1002/jbmr.2422.

15. Wintges K, Beil FT, Albers J, et al. Impaired bone formation and increased osteoclastogenesis in mice lacking chemokine (C-C motif) ligand 5 (Ccl5). J Bone Miner Res 2013; 28: 2070-80.

16. Bourque WT, Gross M, Hall BK. Expression of four growth factors during fracture repair. Int J Dev Biol 1993; 37: 573-9.

17. Hankemeier S, Grassel S, Plenz G, Spiegel HU, Bruckner P, Probst A. Alteration of fracture stability influences chondrogenesis, osteogenesis and immigration of macrophages. J Orthop Res 2001; 19: 531-8.

18. Alexander KA, Chang MK, Maylin ER, et al. Osteal macrophages promote in vivo intramembranous bone healing in a mouse tibial injury model. J Bone Miner Res 2011; 26: 1517-32.

19. Raggatt LJ, Wullschleger ME, Alexander KA, et al. Fracture healing via periosteal callus formation requires macrophages for both initiation and progression of early endochondral ossification. Am J Pathol 2014; 184: 3192-204. 